\title{
Underwater Acoustic Navigation with the WHOI Micro-Modem
}

\author{
Sandipa Singh*, Matthew Grund*, Brian Bingham ${ }^{\dagger}$, Ryan Eustice $^{\ddagger}$, Hanumant Singh*, Lee Freitag* \\ ${ }^{*}$ Woods Hole Oceanographic Institution \\ Woods Hole, MA 02543 USA \\ $\dagger^{\dagger}$ Franklin W. Olin College of Engineering \\ Needham, MA 02492 USA \\ $\ddagger$ Johns Hopkins University \\ Baltimore, MD 21218 USA
}

\begin{abstract}
The WHOI Micro-Modem is a compact, low-power acoustic transceiver that can provide both acoustic telemetry and navigation. Its size and versatility make it ideal for integration in autonomous underwater vehicles (AUVs). The modem supports the use of both broadband and narrowband transponders for long baseline navigation systems, has a modem-to-modem ranging capability, and can be configured to provide synchronous oneway ranging, when integrated with a precision clock. This paper gives an overview of the different navigation systems supported by the Micromodem and presents the results from field tests conducted on the SeaBED AUV in deployments in Greece, the Bluefin AUV, and whale localizations in the Stellwagen Bank Marine Sanctuary.
\end{abstract}

\section{INTRODUCTION}

Acoustic navigation is a vital aspect of underwater vehicle operations. The WHOI Micro-Modem, in addition to providing high-rate underwater acoustic communications, also provides signaling which is useful to an underwater vehicle's navigation system. It can be commanded to interrogate both narrowband and broadband transponders, and provides travel time information. These travel times can then be used to derive vehicle position. In addition, the modem has a one-way ranging capability derived from every communications packet, when provided with an external clock reference, providing an efficient telemtry and ranging solution.

This paper can be broadly divided into two sections. Section II gives an overview of the system software, focusing on the detector. It then goes on to briefly describe narrowband and broadband LBL navigation, ranging and synchronous navigation. Section III will deal with results from various recent experiments.

\section{SYSTEM DESCRIPTION}

The Micro-Modem's real-time embedded operating system is implemented on a low-power fixed-point digital signal processor, the Texas Instruments TMS320C5416 DSP. When receiving, the analog input signal is sampled with a 12-bit A/D converter. Passband data are demodulated and then passed to a matched-filter detector. The detector can be configured to process four separate signals simultaneously within the same band. The detection scheme uses two configurable thresholds. The noise threshold is used to reject impulse noise, while the detection threshold is used to detect a peak from the matched filter output which is normalized by the broadband noise power

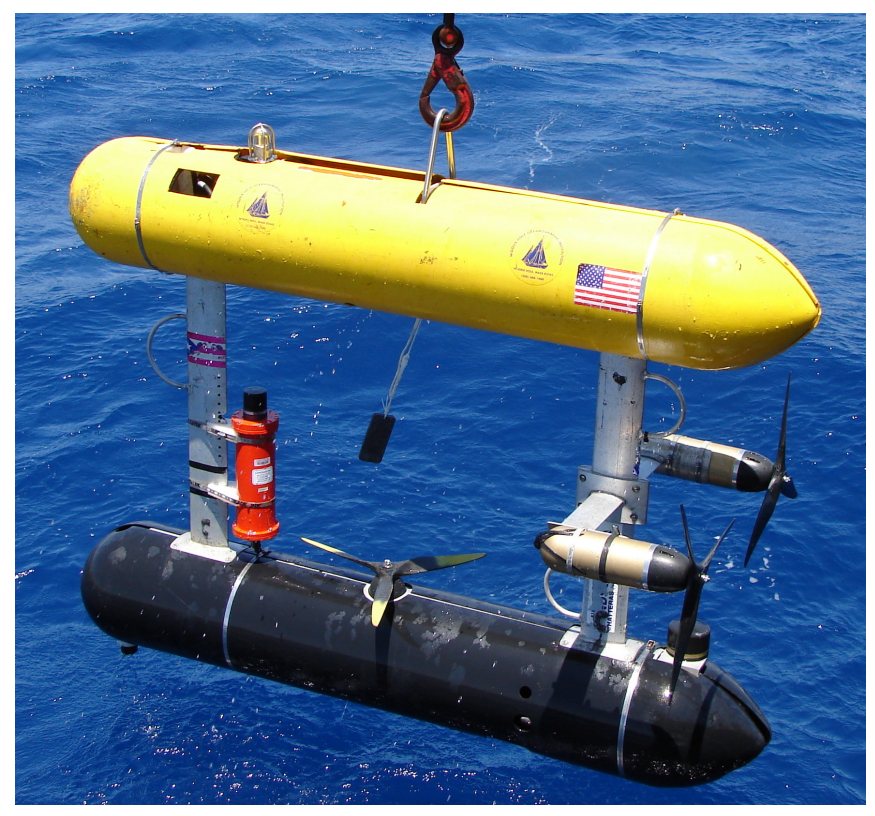

Fig. 1. The SeaBED AUV, developed at Woods Hole Oceanographic Institution, uses the Micro-Modem for telemetry and navigation. It currently can perform narrow-band LBL ranging, and acoustic telemetry synchronous ranging, which is used to correct its DVL asssisted dead-reckoning. SeaBED is designed to make high resolution photo mosaics, do multi-beam bathymetry and also carries a suite of chemical and oceanographic sensors.

estimate. This detector can be configured for a wide variety of signals that can be used to support several navigation schemes. The detector resolution is 125 microseconds.

All communications packets in the Micro-Modem are preceded by an FM sweep of $10 \mathrm{~ms}$ duration and a bandwidth of $4 \mathrm{kHz}$. Table II-B shows some performance metrics for the sweep in differing environments.

\section{A. Broad band REMUS-based LBL navigation}

The Micro-Modem can be used to interrogate up to four REMUS transponders at center frequencies of $24 \mathrm{kHz}$ and $25 \mathrm{kHz}$, using broadband PSK codes. On receiving replies, it publishes one-way travel times which can be used to calculate position using an active navigation solution. The interrogation frequency is $26 \mathrm{kHz}$ at a bandwidth of $4 \mathrm{kHz}$. 
TABLE I

Observed Detector Performance in Various Undersea ENVIRONMENTS TO-DATE

\begin{tabular}{|ccc|}
\hline Center frequency & Channel (depth) & Range \\
\hline $10 \mathrm{KHz}$ & $200 \mathrm{~m}$ shelf & $3.2 \mathrm{~km}$ \\
$15 \mathrm{KHz}$ & $2200 \mathrm{~m}$ offshore & $3.6 \mathrm{~km}$ \\
$25 \mathrm{KHz}$ & $10 \mathrm{~m}$ Very shallow water & $2 \mathrm{~km}$ \\
$25 \mathrm{KHz}$ & 3m Surf Zone & $800 \mathrm{~m}$ \\
\hline
\end{tabular}

\section{B. Narrow-band LBL navigation}

The Micro-Modem also supports narrow-band transponders which are used by the SeaBED, JASON and ABE vehicles. It can be tuned to frequencies ranging from $10 \mathrm{kHz}$ to 30 $\mathrm{kHz}$, depending on the transponders' characteristics. It can be also configured to listen silently without interrogation using an external reference for calculating travel times.

The travel-times measured by the modem can be used to calculate position using active as well as passive navigation schemes [1]. Active navigation uses fixed transponder locations as well as range from the unknown position to each transponder. With three or more range nodes, a linear least squares estimator is used. However with two nodes, the fix is ambiguous and external information is utilized to choose between two possible solutions. The passive navigation scheme uses time-difference of arrivals and transponder positions to solve a linear system of equations for the unknown position. This solution can be iteratively refined using a system based on Taylor series expansion. With four or more nodes, a unique solution can be obtained, but with three nodes, external information is needed to constrain the solution.

\section{Modem to Modem Ranging}

The modem uses the ping command to range to any other node. The ping, which is a short communications packet of approximately 1 second duration, is sent out to the specified node, which sends a return ping after a fixed amount of time, thus enabling the originating node to calculate range.

\section{Synchronous Telemetry and Ranging}

The Micro-Modem provides precise arrival times of communication packets utilizing a user-supplied Pulse-Per-Second (PPS) reference clock signal [2]. The Micro-Modem uses its onboard oscillator to make timing measurements for packet arrivals between the PPS ticks. The packet itself contains the originating node's position information which is used by the vehicle to estimate its position. The message format for the data packet follows the Compact Control Language specifications [3]. It consists of 32 bytes of data containing a high resolution latitude and longitude position fix, the time of that fix and the time of the packet transmission.

Synchronized clocks can also be exploited to calculate oneway range. Synchronous transmission allows the modem to transmit coincident with the PPS signal so that the arrival time on a receiver with a synchronized clock can be used for one-way ranging. With synchronous navigation, multiple vehicles deployed in a network can simultaneously calculate navigation fixes without the degradation associated with timedivision multiplexing of two-way transponder networks.

\section{RESUltS}

This section presents results of several experiments in which the Micro-Modem was succesfully used to provide communication and navigation capabilities. In this paper, we will focus on the navigation data from these experiments.

\section{A. LBL navigation with SeaBed}

The WHOI Micro-Modem has been installed on the SeaBed AUV, shown in figure 1, which was recently deployed off the coast of Andros, Greece on an archaeological cruise. The Micro-Modem was used by the vehicle with two standard Benthos narrow-band transponders with pulses centered at 10.5 and $11 \mathrm{kHz}$ with a duration of $10 \mathrm{~ms}$. Range data thus collected was used to calculate a position fix for the vehicle using an active navigation solution. Figure 2 shows range data from the ship-mounted (topside) modem to the transducer. Predicted data is the estimated range based on the GPS locations and the estimated transponder location. Residual error in range, calculated from GPS logs, is 1.59 $\mathrm{m}$. Figure 3 shows similar data for the $10.5 \mathrm{kHz}$ transponder with an average residual error of $1.11 \mathrm{~m}$.

Figure 4 shows tracking data for the vehicle during a mission off the coast of Andros that lasted 3.5 hours and was carried out at an average depth of 60 meters. It covered an area approximately $2000 \mathrm{~m} \times 1500 \mathrm{~m}$. The blue tracklines show unfiltered LBL data calculated using one-way travel times between the vehicle and the two transponders. The red line is Doppler Velocimeter (DVL) data used in conjunction with a 3 axis north-seeking (IXSEA Octans) fibre-optic gyro (FOG) system. As expected, this solution tends to drift over time. Typically, LBL data will be filtered and fused with the DVL-FOG estimate to obtain absolute position with both high precision and accuracy [4].

\section{B. Synchronous ranging with SeaBed}

During recent sea trials of the SeaBed vehicle with the R/V Tioga near Wood Hole in shallow water, synchronous communications with the Micro-Modem were tested from the vehicle to the ship (topside). All acoustic communications traffic between the topside modem and vehicle modem originated on a PPS clock edge. Accurate synchronization of the modem clock with a GPS receiver enabled both the vehicle and the topside modem to calculate precise one-way ranges while receiving vehicle data through the acoustic packets at the same time. Ground truthing with a LINKQUEST USBL system showed the one-way travel time errors to be of the order of $1 \mathrm{~ms}$. The onboard drift of the PPS clock was $1 \mu \mathrm{s}$. These ranges can be used along with DVL-FOG estimate to generate position estimates that can be combined with vehicle LBL navigation for higher precision and accuracy [5]. Synchronous communications can thus eliminate the need for deployment of transponders for vehicle navigation. 

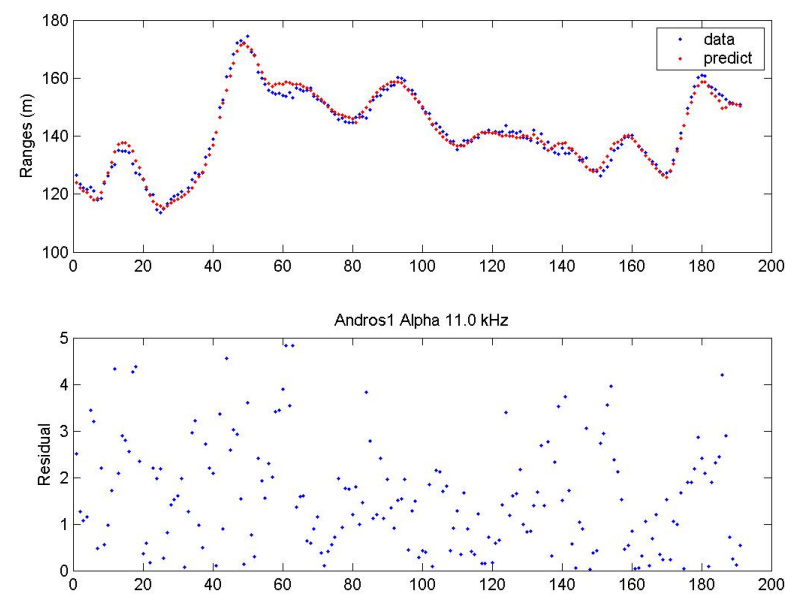

Fig. 2. Range data from the topside modem to the Alpha $(11 \mathrm{kHz})$ transducer plotted over time, in minutes. Predicted ranges calculated from GPS logs. Average residual error in range is $1.59 \mathrm{~m}$
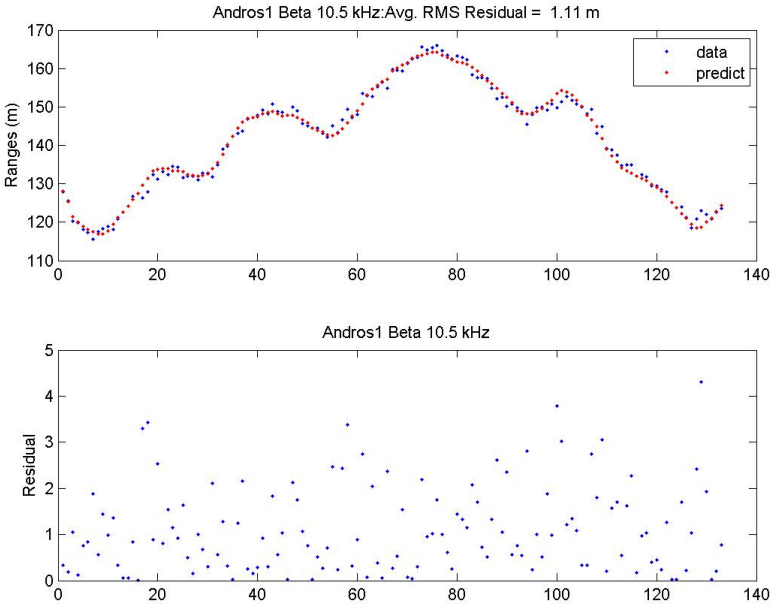

Fig. 3. Range data from the topside modem to the Beta $(10.5 \mathrm{kHz})$ transducer plotted over time, in minutes. Predicted ranges calculated from GPS logs. Average residual error in range is $1.11 \mathrm{~m}$

\section{Synchronous ranging with Bluefin $A U V$}

Synchronous communications have been used by Bluefin's AOFNC-1 vehicle to augment tracking data from dead reckoning and GPS. Figure 7 shows test results from a single run of the vehicle between two transducers mounted $1.5 \mathrm{~m}$ below two kayaks, labeled K0 and K1. The kayaks defined a baseline for the vehicle which is about $140 \mathrm{~m}$ long. Ranges obtained from synchronous communications were used to filter deadreckoned trajectory data, initialized with GPS at the surface. It can be seen that the range filtered data agrees with GPS data better than the unfiltered, dead-reckoned tracks.

Eventually, one-way travel time data from the modem will be used by the vehicle as part of a moving LBL navigation system in which the kayaks will be moving in the water

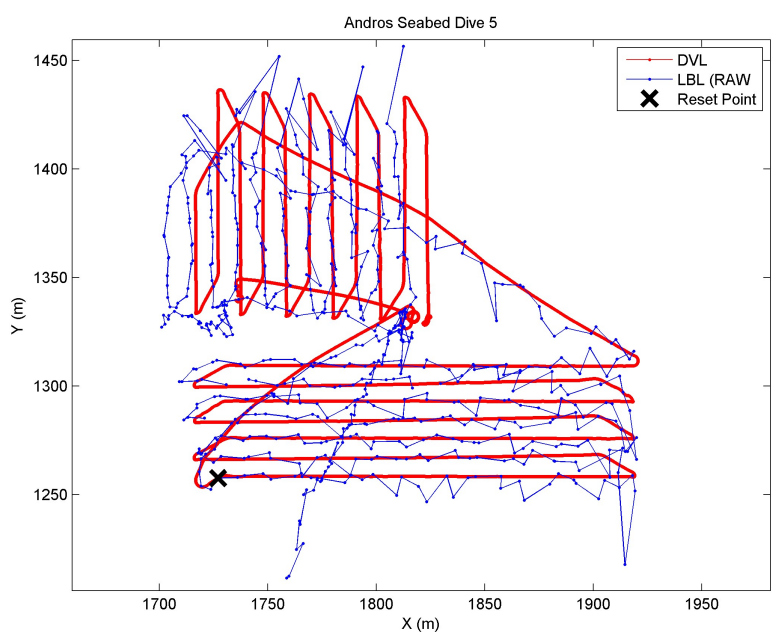

Fig. 4. SeaBed LBL navigation: Blue lines show raw tracking data from the modem using ranges from 2 transponders. Red lines are obtained from the Doppler Velocitimeter and show considerable drift over time.

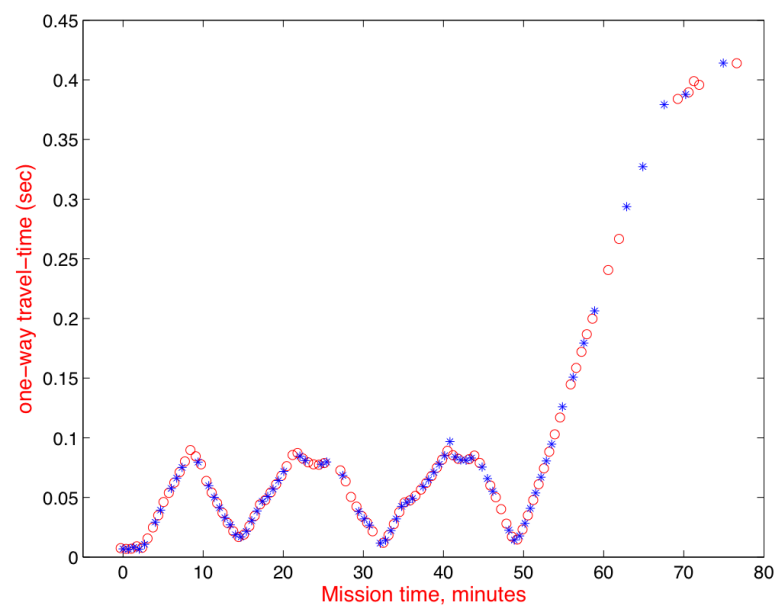

Fig. 5. Synchronous arrival times from the SeaBed AUV. The blue stars are vehicle travel times and the red circles are travel times recorded by the topside.

simultaneously with the vehicle.

\section{Whale tracking and localization}

Passive navigation is a useful technique for tracking and localizing marine mammals. The WHOI Micro-Modem has been used to successfully track a humpback whale in the Stellwagen Bank National Marine Sanctuary using the Realtime Acoustic Tracking System (RATS) developed for Mark Baumgartner of the Woods Hole Oceanographic Institution. This consists of four buoys, each equipped with a Micro-Modem listening passively for a $36 \mathrm{kHz}$ pulse from a pinger that is attached to the whale via a suction cup. The pinger has a pressure sensor, and it varies the time between pings in proportion to the depth. Using pseudo ranges from the buoys and absolute depth values from the pinger, a position estimate for the whale 


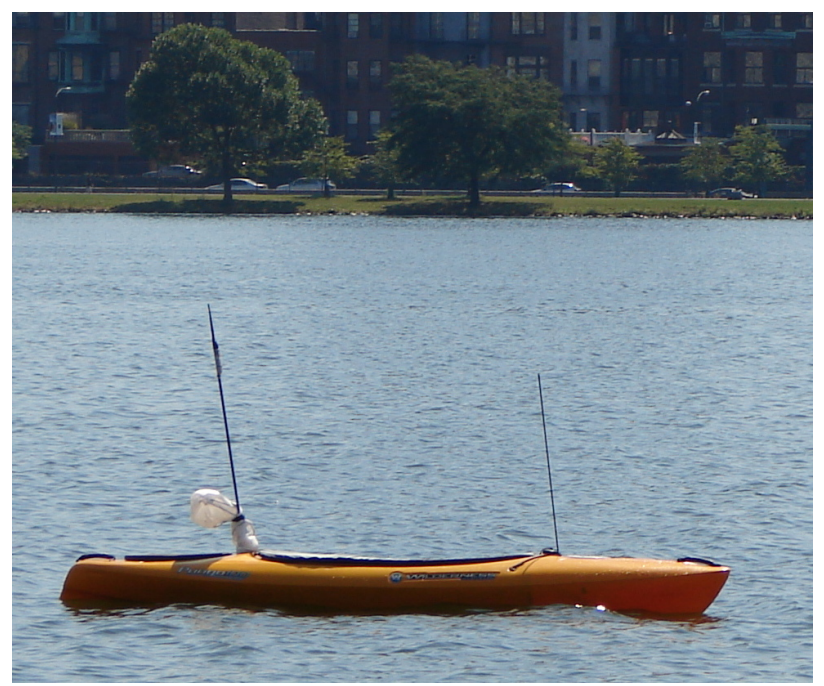

Fig. 6. A kayak autonomous surface craft (ASC), developed jointly by MIT and Robotic Marine Systems, was used in a navigation aid role, to provide synchronous navigation and ranging telemetry, using the WHOI Micro-Modem.

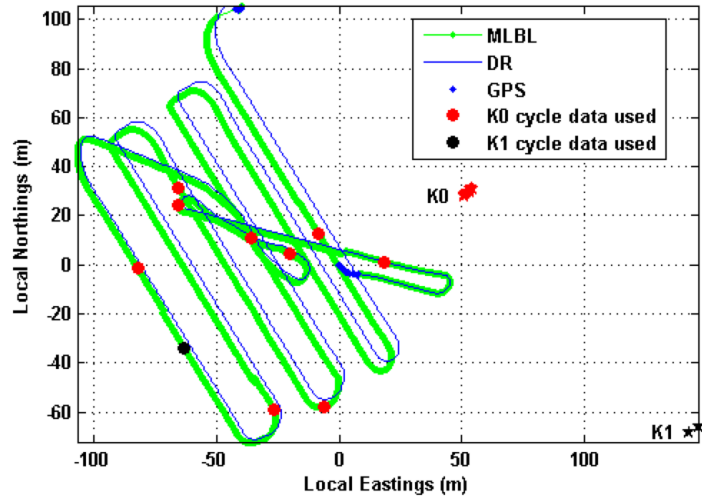

Fig. 7. Bluefin AUV AOFNC1 tracks: Dead reckoning tracks in blue are overlaid by green tracks, filtered by one-way travel time data. Red circles indicate range fix from K0, while black circles indicate range fixes from K1.

in $\mathrm{x}, \mathrm{y}$ and $\mathrm{z}$ can be arrived at using the passive navigation scheme described in section II. The buoys also carry a GPS and freewave modem so that detections and buoy positions are reported in real time. This allows the observer to get close to the animal even when it is submerged. Figure 8 shows 1 hour of tracking data collected on August 30, 2005 1800-1900 UTC on the humpback whale. Three distinct dives are clearly visible during this tracking period.

\section{CONCLUSION}

The WHOI Micro-Modem is being widely used by a variety of vehicles as a low-power acoustic communication and navigation system. Its compact size and low power consumption makes it ideal for integration with AUVs. Future work includes higher rate communication packets for synchronous communications, networking capabilities and configurable power

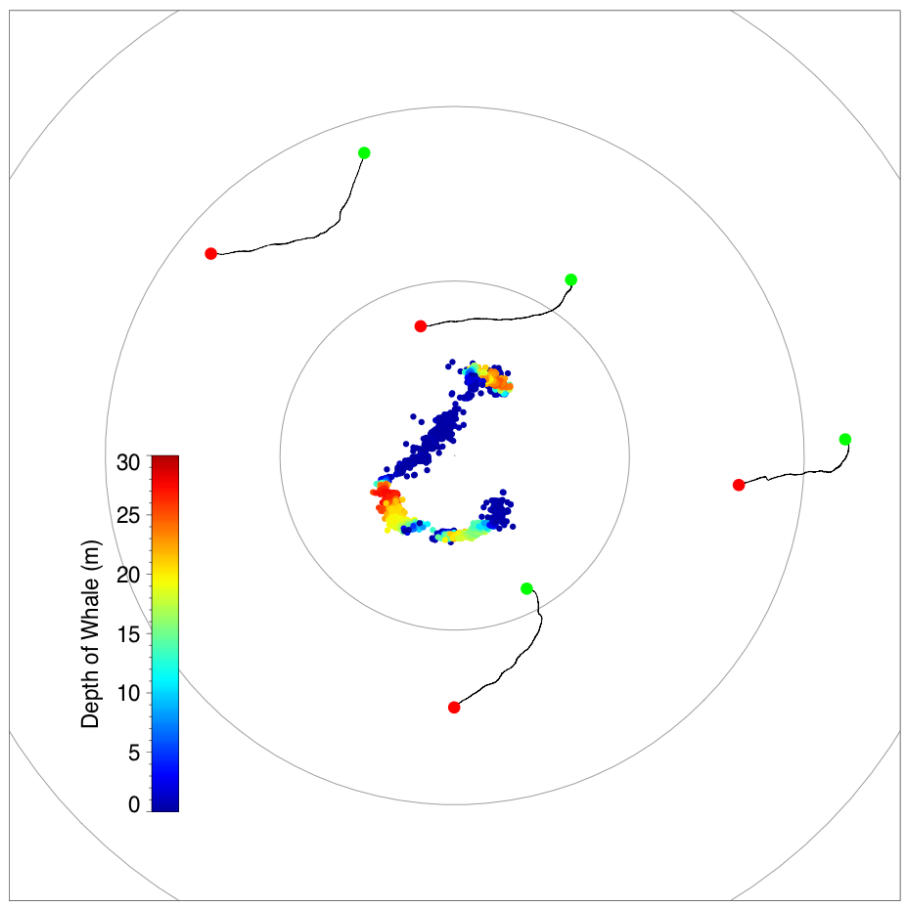

Fig. 8. Humpback whale tracking data. Estimated whale positions are shown as colored dots with the color indicating the depth of the animal. The gray concentric circles are 500 meters apart. The lines indicate the trajectories of the 4 tracking buoys over the 1-hour period. The green and red filled circles indicate the positions of the buoys at the beginning and end of the period, respectively.

control.

\section{ACKNOWLEDGEMENTS}

The authors would like to thank Mark Baumgartner of the Woods Hole Oceanographic Institution for providing whale tracking data and Jerome Vaganay of Bluefin Robotics, Cambridge Massachusetts, for providing synchronous ranging data from the AOFNC-1 vehicle. The SeaBED portion of this work was made possible by a grant from NSF under grant number 99868321.

\section{REFERENCES}

[1] L. Freitag, M. Johnson, M. Grund, S. Singh, and J. Preisig, "Integrated Acoustic Communication and Navigation for Multiple UUVs," in IEEE Proceedings of Oceans, Honolulu, October 2001, pp. 2065-2070.

[2] L. Freitag, M. Grund, J. Partan, S. Singh, P. Koski, and K. Ball, "The WHOI Micro-Modem: An Acoustic Communications and Navigation system for Multiple Platforms," in Proc. Oceans 2005, Sept. 2005.

[3] R. Stokey, "A Compact Control Language for Autonomous Underwater Vehicles," Woods Hole Oceanographic Institution, Tech. Rep., 2005.

[4] L. Whitcomb, D. Yoerger, H. Singh, and D. Mindell, "Towards precision robotic maneuvering, survey and manipulation in unstructured undersea environments," Robotics Research - The Eighth International Symposium, pp. 45-54, 1998.

[5] H. Singh, J. Bellingham, F. Hover, S. Lerner, B. Moran, K. von der Heydt, and D. Yoerger, "Docking for autonomous ocean sampling network," IEEE Journal of Oceanic Engineering, vol. 26, no. 4, pp. 498-514, 2001. 